\title{
Anti-CD38 autoantibodies: Characterisation in new-onset Type I diabetes and latent autoimmune diabetes of the adult (LADA) and comparison with other islet autoantibodies
}

\author{
R. Mallone 1 , E. Ortolan ${ }^{2}$, S. Pinach ${ }^{1}$, M. Volante ${ }^{3}$, M.M. Zanone ${ }^{1}$, G. Bruno ${ }^{1}$, G. Baj ${ }^{4}$, T. Lohmann , \\ P. Cavallo-Perin ${ }^{1}$, F. Malavasi ${ }^{2}$ \\ ${ }^{1}$ Department of Internal Medicine, University of Torino, Torino, Italy \\ ${ }^{2}$ Laboratory of Immunogenetics, Department of Genetics, Biology and Biochemistry, University of Torino, Torino, Italy \\ ${ }^{3}$ Department of Biomedical Sciences and Oncology, University of Torino, Torino, Italy \\ ${ }^{4}$ Laboratory of Gyneacological Oncology, Department of Medical Sciences, University of Eastern Piedmont “A. Avogadro", \\ Novara, Italy \\ ${ }^{5}$ Department of Internal Medicine I, University of Erlangen, Erlangen, Germany
}

\begin{abstract}
Aims/hypothesis. Serum anti-CD38 autoantibodies (aAbs) have been reported in 17 to $19 \%$ of patients with long-standing Type I (insulin-dependent) diabetes mellitus and Type II (non-insulin-dependent) diabetes mellitus. Whether these aAbs are also found in new-onset Type I diabetes and in Latent Autoimmune Diabetes in Adults (LADA) is not known, as is their relationship with conventional islet aAbs.

Methods. These issues were addressed by studying new-onset Type I and LADA diabetic cohorts with a recently developed anti-CD38 enzymatic immunoassay.

Results. Anti-CD38 aAb prevalence among newonset Type I patients (3.8\%) was lower than previously found in long-standing Type I diabetes $(11.7 \%$, as defined with the 97.5 percentile cutoff; $p=0.01$ ), suggesting a late appearance of these aAbs. Among LADA patients, $14.9 \%$ were anti-CD38+. Anti-CD38 were only associated with anti-GAD aAbs in newonset Type I diabetes. Although the CD38 target mol-
\end{abstract}

ecule was expressed in human pancreatic islets, antiCD38 aAbs did not contribute to the islet cell antibody (ICA) immunofluorescence reactivity. All the positive sera analysed for $\mathrm{Ca}^{2+}$ release were found to mobilise it. In agreement with these agonistic features, anti-CD38+ new-onset Type I patients showed higher fasting C-peptide values as compared to negative counterparts; the association was stronger when the analysis was limited to the agonistic anti-CD38+ sera. A similar trend was found among LADA patients.

Conclusion/interpretation. Anti-CD38 aAbs are distinct markers of islet autoimmunity which are more prevalent in long-standing disease, as opposed to the other known islet aAbs. Their in vitro agonistic properties could be operating in vivo as well, as they identify sub-groups of patients with higher residual betacell function. [Diabetologia (2002) 45:1667-1677]

Keywords ADP-ribosyl cyclase, autoimmunity, beta cell, calcium, ectoenzymes, GAD, ICA, islet cell antibodies.
Received: 20 March 2002 / Revised: 28 June 2002

Published online: 24 October 2002

(C) Springer-Verlag 2002

Corresponding author: R. Mallone, MD, Department of Internal Medicine, Corso AM Dogliotti, 14, 10126 Torino, Italy. E-mail: roberto.mallone@unito.it

Abbreviations: aAbs, Autoantibodies; AU, arbitrary units; $\left[\mathrm{Ca}^{2+}\right]_{\mathrm{i}}$, intracellular $\mathrm{Ca}^{2+}$ concentration; EIA, enzymatic immuno-assay; ICA, islet cell antibodies; LADA, Latent Autoimmune Diabetes in Adults; mAb, monoclonal antibody; rsCD38, recombinant soluble CD38.
Damage to the beta cell is one of the crucial events leading to diabetes. Whether this damage occurs by autoimmune mechanisms, as in Type I (insulin-dependent) diabetes mellitus [1], or by other less defined phenomena (e.g., glucose toxicity [2], lipotoxicity [3], prolonged excessive insulin secretion [4]), as in Type II (non-insulin-dependent) diabetes mellitus [5], the final result is impaired insulin secretion [6]. A number of autoantibodies (aAbs) marking the immune-mediated destruction of the beta cell typical of Type I diabetes have been described since the identification of islet cell 
antibodies (ICA) [7]. ICA comprise a number of autoreactive immunoglobulins with different target antigens, one of which has later been identified as GAD [8, 9]. As for ICA, anti-GAD reactivity is higher at diagnosis but, unlike ICA, it persists for a long time in sera, thus achieving high sensitivity in identifying diabetic patients [10]. Anti-IA-2 aAbs are responsible for another major subfraction of the ICA reactivity $[8,11]$ and, together with anti-GAD aAbs, they account for about $90 \%$ of ICA immunofluorescence [8]. Anti-IA-2 aAbs are an early marker of islet autoimmunity, being mainly found at disease onset $[8,11]$; they are also found with higher frequency in younger patients [12] independently of disease duration [13], probably reflecting a severe beta-cell destruction leading to the clinical onset of the disease early in life $[14,15,16$, 17]. Although these markers are mainly found in Type I diabetic patients, a subgroup of patients routinely classified as Type II was also found to show high prevalences of ICA and anti-GAD, but not of anti-IA-2 aAbs. These patients have later been classified as having a slow progressive form of Type I diabetes, defined as Latent Autoimmune Diabetes of the Adult (LADA) $[12,13,18,19,20,21,22]$.

Anti-CD38 aAbs have been described as new markers of beta-cell autoimmunity [23, 24, 25]. The CD38 target molecule is a membrane glycoprotein mainly expressed in the haematological compartment, functioning as a signalling receptor and an ectoenzyme capable of producing and degrading cyclic ADP-ribose (cADPR), a $\mathrm{Ca}^{2+}$-mobilising second messenger $[26,27]$. cADPR produced by CD38 has been implicated in the mechanisms of insulin secretion in rodents $[28,29]$. We have recently developed an antiCD38 enzymatic immuno-assay, suitable for screening large cohorts of patients [25]. With this approach, prevalences of $19.1 \%$ and $16.7 \%$ were found among Caucasian Type I and Type II diabetic subjects with long-standing disease, respectively [25]. The majority of these aAbs showed agonistic properties, capable of triggering $\mathrm{Ca}^{2+}$ release from CD38+ target cells [25]. Anti-CD $38^{+}$sera incubated with purified islet cells induced not only $\mathrm{Ca}^{2+}$ mobilisation, but also insulin secretion, with a direct correlation between the two effects $[24,30]$. In agreement with these experimental findings, anti-CD $38^{+}$Type II diabetic patients were characterized by higher residual beta-cell function and higher BMI, i.e., a phenotype opposite to that of LADA [25].

On these grounds, we aimed to answer three critical questions: (i) are anti-CD38 aAbs more prevalent at Type I diabetes onset than in late stage disease like the other known islet aAbs (ii) despite the phenotype opposite to that of LADA observed in anti-CD38+ Type II patients [25], are anti-CD38 aAbs also found among LADA subjects (iii) what is the relationship, if any, between anti-CD38 aAbs and the conventional markers of beta-cell autoimmunity. These issues were addressed by studying a large cohort of newly diagnosed Type I diabetic patients and a smaller group of LADA patients. Comparisons between anti-CD38 and the other islet aAbs were also carried out, both in these study cohorts and in a previously characterised group of long-standing Type I diabetic subjects [25].

\section{Subjects and methods}

Patients. We recruited 188 Caucasian new-onset Type I diabetic subjects from patients identified by the population-based registry of the province of Torino [31]. Blood samples were obtained within two months after onset. Diagnosis of Type I diabetes was made according to the American Diabetes Association and WHO criteria [32, 33]. We selected 67 Caucasian LADA subjects with the following inclusion criteria: age more than 30 years old at diagnosis, no ketoacidosis at onset and absence of insulin therapy in the first 6 months after diagnosis, positivity of either anti-GAD or ICA. Exclusion criteria were secondary forms of diabetes and a history of previous interferon therapy. Healthy control subjects with no family history of diabetes, normal fasting plasma glucose and similar sex and age distribution were selected at the local blood bank and at the Department of Paediatrics among children undergoing minor elective surgical procedures. All blood samples were obtained in the morning after overnight fasting. Sera were divided in aliquots and stored at $-80^{\circ} \mathrm{C}$ until analysis. A cohort of Type I diabetic patients previously characterised for anti-CD38 titres [25] was also included and assayed for islet aAbs for comparative analyses. Informed consent was obtained from each participant and the study was approved by the Hospital Review Board and Ethics Committee.

Enzymatic immuno-assay for anti-CD38 aAbs. Anti-CD38 aAb titres were measured with an enzymatic immuno-assay (EIA), using recombinant soluble CD38 (rsCD38, made available by H. C. Lee, University of Minnesota, Minneapolis, Minn., USA) [34] as the immobilised target antigen [25]. The antiCD38 EIA was previously shown to have a consistent reproducibility (4.3\% intra-assay and $11.6 \%$ inter-assay variability) [25]. EIA plates were coated with rsCD38 and subsequently saturated with $5 \%$ non-fat dry milk in PBS. Sera of patients diluted 1:3,000 were added and incubated for $2 \mathrm{~h}$ at $37^{\circ} \mathrm{C}$. This dilution was chosen as the one effectively differentiating between positive and negative samples, as compared with the 1:640 previously used [25], minimising aspecific binding to the EIA plate; the previously characterised cohort of Type I patients was also reanalysed at this higher dilution. After washing, an alkaline phosphatase-conjugated anti-human Ig monoclonal antibody (mAb) (Sigma, St. Louis, Mo., USA) was added; no difference was observed when this was substituted by an anti-human $\operatorname{IgG} \mathrm{mAb}$. The reaction was visualised with paranitrophenylphosphate chromogen and reading of the absorbance at $405 \mathrm{~nm}$ after $45 \mathrm{~min}$. Each sample was assayed in triplicate in at least two different experiments and the mean of these six measurements was used for all subsequent calculations. A standard curve was derived from a highly reactive serum in each experiment: the optical density (OD) obtained with this serum was conventionally defined as equal to 10000 arbitrary units $(\mathrm{AU} / \mathrm{ml})$ and the reactivity of the other samples calculated in proportion with the following formula:

$\mathrm{AU} / \mathrm{ml}=\frac{(\text { sample OD }- \text { negative control OD }) \times 10,000}{\text { standard serum OD }- \text { negative control OD }}$ 
where the negative control was obtained by incubation of the immobilised rsCD38 with secondary $\mathrm{mAb}$ alone. The upper limit of the normal range was defined as the 97.5 centile of the anti-CD38 titres measured in the sera of 358 control healthy subjects (median age 17 , range $0-95$ years). This percentile was chosen as the one effectively differentiating between diabetic patients and control subjects; the cut-off value thus obtained was $6927 \mathrm{AU} / \mathrm{ml}$. Each study group was compared with a cohort of healthy control subjects matched for age and sex.

Islet $a A b$ assays. ICA were assayed by indirect immunofluorescence on frozen sections of human blood group 0 pancreas and positivity expressed in Juvenile Diabetes Foundation (JDF) units, by a standard curve based on the international JDF unit reference sample. An ICA titre more than or equal to five JDF units was considered as positive. In preadsorption experiments, sera were preincubated with saturating amounts $(1 \mu \mathrm{g}$ in $10 \mu$ of each serum) of either rsCD38 or an irrelevant protein (HIV gp120; Chiron, Emeryville, Calif., USA) prior to ICA testing. Anti-GAD aAbs were measured by a radioligand assay using human recombinant GAD65 as the target antigen (RSR, Cardiff, UK) [35]. Sera were incubated with 125I-labelled GAD65, followed by the addition of solid phase protein A to precipitate the labelled immune complexes; after centrifugation, supernatants were discarded and the precipitates counted for ${ }^{125} \mathrm{I}$. Samples were classified as positive for anti-GAD titres more than or equal to $0.9 \mathrm{U} / \mathrm{ml}$. Anti-IA-2 aAb titres were measured by a similar radioligand assay with the same procedure, using human recombinant IA-2 as the target antigen (RSR). A titre more than or equal to $0.75 \mathrm{U} / \mathrm{ml}$ was classified as positive. A $100 \%$ sensitivity and specificity in these three islet $\mathrm{aAb}$ assays was repeatedly achieved by our Laboratory in the Immunology of Diabetes Workshop Proficiency Programs.

Immunohistochemistry. Tissues were fixed in $10 \%$ buffered formalin and embedded in paraffin. Sections were collected on poly-L-lysine-treated slides, dewaxed, rehydrated and immersed in $3 \% \mathrm{H}_{2} \mathrm{O}_{2}$ to quench endogenous peroxidase activity. Slides were then immersed in $10 \mathrm{mmol} / \mathrm{l}$ sodium citrate buffer, $\mathrm{pH}$ 6.0, and heated three times for $3 \mathrm{~min}$ at high setting in a $750 \mathrm{~W}$ output microwave oven. An incubation for $15 \mathrm{~min}$ with an avidin-blocking reagent (Biogenex, San Ramon, Calif., USA) at room temperature was then done to prevent endogenous biotin reactivity. The slides were subsequently incubated for $30 \mathrm{~min}$ at room temperature with the AT1 anti-CD38 mAb (provided by J. Hilgers, BioProbe BV, Amstelveen, The Netherlands). The reaction was visualised with the Dako LSAB2 peroxidase kit (Dako, Glostrup, Denmark) and liquid $\mathrm{H}_{2} \mathrm{O}_{2}-3-3^{\prime}$-diaminobenzidine (Sigma). In preadsorption experiments, the AT1 mAb was preincubated overnight at $4^{\circ} \mathrm{C}$ with saturating amounts of either rsCD38 or the gp120 irrelevant protein. For indirect immunofluorescence, frozen pancreatic tissue sections were reacted with IB4 $\left(\operatorname{IgG}_{2 \mathrm{a}}\right)$, OKT10 $\left(\operatorname{IgG}_{1}\right)$, T16 (IgG ${ }_{1}$; Immunotech, Marseille, France), $\mathrm{HB} 7\left(\mathrm{IgG}_{1}\right)$ and AT1 $\left(\mathrm{IgG}_{1}\right)$ anti-CD38 mAbs followed by fluorescein isothiocyanate- or phycoerithrin-conjugated anti-mouse $\mathrm{Ig}$ secondary Ab.

Agonistic activity of anti-CD38 aAbs on $\mathrm{Ca}^{2+}$ release. Analysis of $\mathrm{Ca}^{2+}$ mobilisation mediated by anti-CD38 aAbs was carried out on the Jurkat $\mathrm{T}$ acute lymphoblastic leukaemia cell line [25]. Cells were loaded with Fluo 3-AM (Sigma), a Ca ${ }^{2+}$ sensitive fluorescent dye and subsequently incubated with $50 \mu \mathrm{l}$ of serum. Effects on intracellular $\mathrm{Ca}^{2+}$ concentration $\left(\left[\mathrm{Ca}^{2+}\right]_{\mathrm{i}}\right)$ were continuously analysed at $37^{\circ} \mathrm{C}$ with a FACSort cytofluorimeter (Becton Dickinson, Milano, Italy). Controls included sera from diabetic patients negative by anti-CD38 EIA and cells treated with the 4-bromo A23187 ionophore (Sigma) to ensure proper Fluo 3-AM loading. Modifications over time of $\left[\mathrm{Ca}^{2+}\right]_{i}$ were followed by continuously recording fluorescence shifts of Fluo 3-AM during a 5-min time-course; the extrapolated Fluo 3-AM mean fluorescence intensity was then used for numerical presentation of data. The cut-off was set at the value for which both sensitivity and specificity of the assay were of $100 \%$.

Clinical and metabolic parameters. $\mathrm{HbA}_{1 \mathrm{c}}$ was measured by high-performance liquid chromatography. Fasting plasma C-peptide was measured by radioimmunoassay (DPC, Los Angeles, Calif., USA). The three therapy categories for LADA subjects (diet alone, oral hypoglycaemic agents and insulin, with or without oral hypoglycaemic agents) were considered mutually exclusive.

Statistical analysis. Values are expressed as means \pm SD or median (range), according to their distribution. Comparisons between proportions were made with the $\chi^{2}$ test and Fisher's exact test, when appropriate. Comparisons of means between two groups were carried out with the Student's $t$ test for normal distributed variables or with the Mann-Whitney $U$ test for nonnormal variables. Analysis of data was done using Stata 7.0 (Stata Corporation, College Station, Tex., USA). Differences were considered statistically significant for $p$ values less than or equal to 0.05 .

\section{Results}

Characteristics of the patients. Clinical and anti-islet autoimmunity profiles of the 183 new-onset Type I diabetic patients studied are shown in Table 1. Their

Table 1. Characteristics of newly diagnosed Type I diabetic patients and matched healthy control subjects

\begin{tabular}{|c|c|c|c|}
\hline & $\begin{array}{l}\text { New-onset Type I diabetic } \\
\text { subjects }(n=183)\end{array}$ & $\begin{array}{l}\text { Control subjects } \\
(n=171)\end{array}$ & $p$ \\
\hline Sex (male and female) & $54.6 \%-45.4 \%$ & $53.5 \%-46.5 \%$ & NS \\
\hline Fasting plasma C-peptide (ng/ml) & $0.44(0-5.70)$ & & - \\
\hline Autoimmune diseases $(\%)$ & $2.7 \%$ & - & - \\
\hline $\mathrm{ICA}^{+}(\%)$ & $56.2 \%$ & - & - \\
\hline
\end{tabular}

Data are expressed as per cent proportion, means \pm SD or median (range), as appropriate 


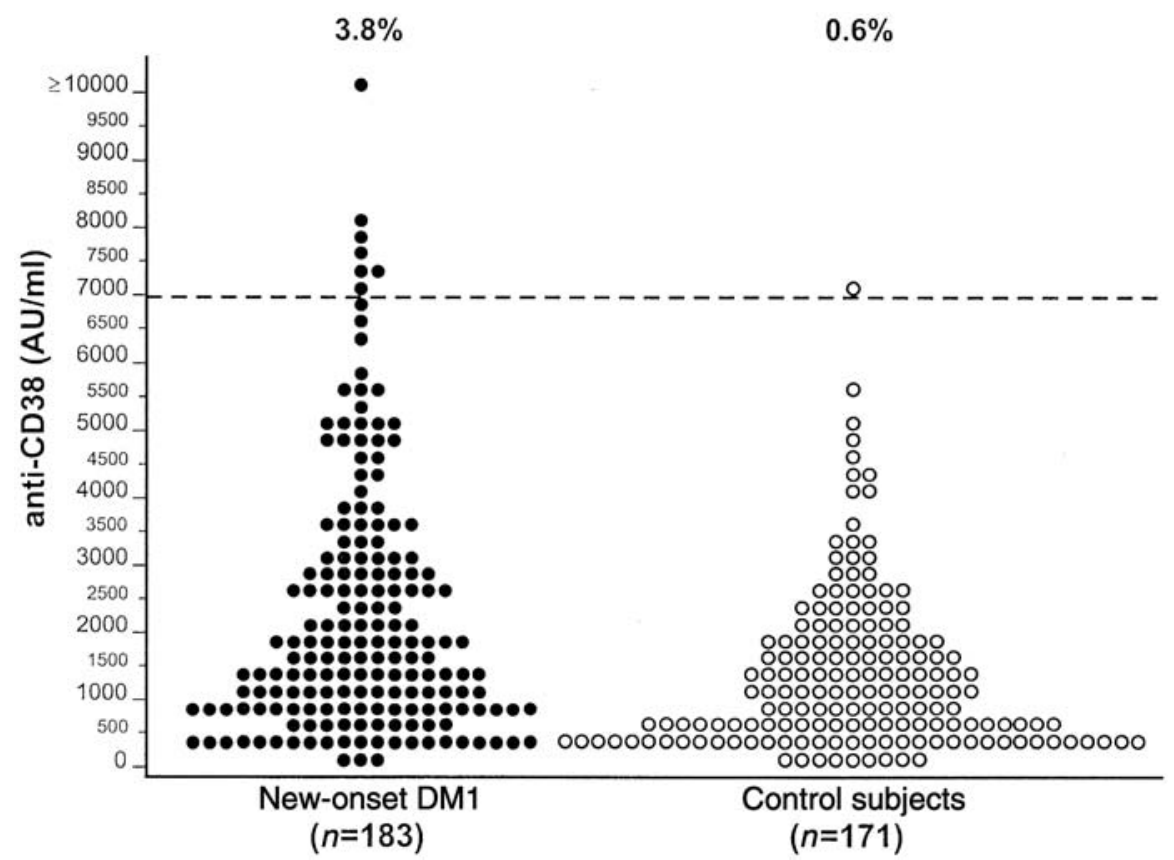

Fig. 1. Anti-CD38 reactivity in sera from new-onset Type I diabetic (DM 1) patients. Values are expressed in $\mathrm{AU} / \mathrm{ml}$; the dotted line indicates the cut-off value (97.5 per centile) of

$6927 \mathrm{AU} / \mathrm{ml}$. The per cent proportion of anti-CD38+ subjects is indicated above each distribution plot. $p=0.04$ for comparison between the two groups

Table 2. Characteristics of LADA patients and matched healthy control subjects

\begin{tabular}{|c|c|c|c|}
\hline & LADA patients $(n=67)$ & Control subjects $(n=66)$ & $p$ \\
\hline Sex (male and female) & $50.7 \%-49.3 \%$ & $56.1 \%-43.9 \%$ & NS \\
\hline Age (years) & $56.4 \pm 12.7$ & $57.9 \pm 14.2$ & NS \\
\hline Diabetes duration (years) & $4(0-33)$ & - & - \\
\hline BMI $\left(\mathrm{kg} / \mathrm{m}^{2}\right)$ & $25.0 \pm 4.0$ & $25.8 \pm 3.6$ & NS \\
\hline $\mathrm{HbA}_{1 \mathrm{c}}(\%)$ & $8.6 \pm 2.4$ & $5.4 \pm 0.5$ & $<0.001$ \\
\hline Autoimmune diseases (\%) & $25.0 \%$ & - & - \\
\hline $\mathrm{ICA}^{+}(\%)$ & $47.8 \%$ & - & - \\
\hline Anti-GAD $+(\%)$ & $95.5 \%$ & - & - \\
\hline
\end{tabular}

Data are expressed as per cent proportion, means \pm SD or median (range), as appropriate. D, diet alone; OHA, oral hypoglycaemic agents; I, insulin (alone or in combination with $\mathrm{OHA}$ ).

mean age was $12.5 \pm 8.2$ years and they had a prevalence of islet aAbs similar to that reported in literature for Type I diabetic patients at diagnosis $[8,36,37,38$, $39,40]$. The characteristics of the 67 LADA patients studied are shown in Table 2. Clinical and autoimmune characteristics of a long-standing Type I diabetic cohort previously characterised for anti-CD38 aAbs [25] are summarised in Table 3 . The prevalence of anti-CD $38^{+}$individuals in this group, as defined with the 97.5 percentile cutoff, was $11.7 \%$.

Titres and prevalence of anti-CD38 aAbs among newonset Type I and LADA diabetic patients. Anti-CD38 $\mathrm{aAb}$ titres obtained for new-onset Type I diabetic subjects are shown in Fig. 1. The distribution shows a continuous pattern spanning through all values; the majority of subjects (both diabetic and healthy) showed reactivities less than $2000 \mathrm{AU} / \mathrm{ml}$. Among the 183 patients studied, 7 (3.8\%) were above the cut-off value selected, whereas only 1 out of the 171 control subjects $(0.6 \%)$ was characterised as anti-CD38+ $(p=0.04)$. The prevalence observed in new-onset Type I patients was lower than the $11.7 \%$ found in a cohort of Type I diabetic patients with long-standing disease (Table 3$)$ using the same assay $(p=0.01)$. Although these two new-onset and long-standing Type I diabetic cohorts had different ages at diagnosis $(12.5 \pm 8.2$ vs $17.3 \pm 9.2$ years, respectively; Table 1 and 3), the anti-CD38 prevalence was lower in the former even after excluding the 40 subjects less than 6 years old in the new-onset Type I group $(4.9 \%$ vs $11.7 \% ; p=0.05)$. 


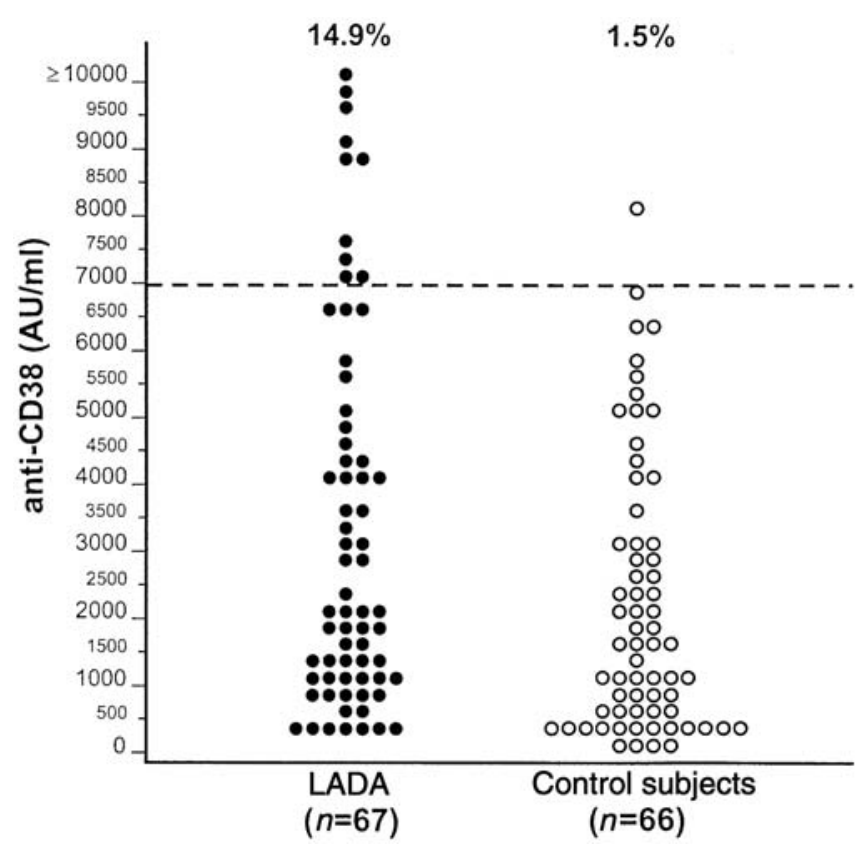

Fig. 2. Anti-CD38 reactivity in sera from LADA patients. Values are expressed in $\mathrm{AU} / \mathrm{ml}$; the dotted line indicates the cut-off value (97.5 centile) of $6927 \mathrm{AU} / \mathrm{ml}$. The per cent proportion of anti-CD38+ subjects is indicated above each distribution plot. $p=0.005$ for comparison between the two groups

Table 3. Characteristics of Type I diabetic subjects, previously characterised for anti-CD38 aAbs

\begin{tabular}{|c|c|}
\hline & $\begin{array}{l}\text { Type I diabetic patients } \\
(n=94)\end{array}$ \\
\hline Sex (male and female) & $54.4 \%-45.6 \%$ \\
\hline Age at diagnosis (years) & $17.3 \pm 9.2$ \\
\hline Diabetes duration (years) & $19.5(1-47)$ \\
\hline $\mathrm{HbA}_{1 \mathrm{c}}(\%)$ & $8.4 \pm 1.5$ \\
\hline Fasting plasma C-peptide (ng/ml) & $0(0-1.68)$ \\
\hline Anti-CD38+ (\%) & $11.7 \%$ \\
\hline $\mathrm{ICA}^{+}(\%)$ & $4.9 \%$ \\
\hline Anti-GAD $+(\%)$ & $35.6 \%$ \\
\hline Anti-IA-2+ $(\%)$ & $5.7 \%$ \\
\hline
\end{tabular}

Data are expressed as per cent proportion, means \pm SD or median (range), as appropriate

Anti-CD38 aAb titres obtained for LADA patients are shown in Fig. 2. The prevalence of anti-CD38+ LADA patients was 10 out of $67,14.9 \%$ as compared to $1.5 \%$ among the 66 control individuals $(p=0.005)$. The prevalence obtained was not different to that observed in the previously studied cohorts of longstanding Type I diabetic patients $(11.7 \%$; Table 3$)$ and Type II diabetic patients $(17.5 \%$, as defined with the 97.5 centile cut-off).

Comparison of anti-CD38 with other islet aAbs. Comparative analyses of anti-CD38 with ICA, antiGAD and anti-IA-2 aAbs were carried out in different diabetic groups (Fig. 3). Positivity for anti-GAD aAbs
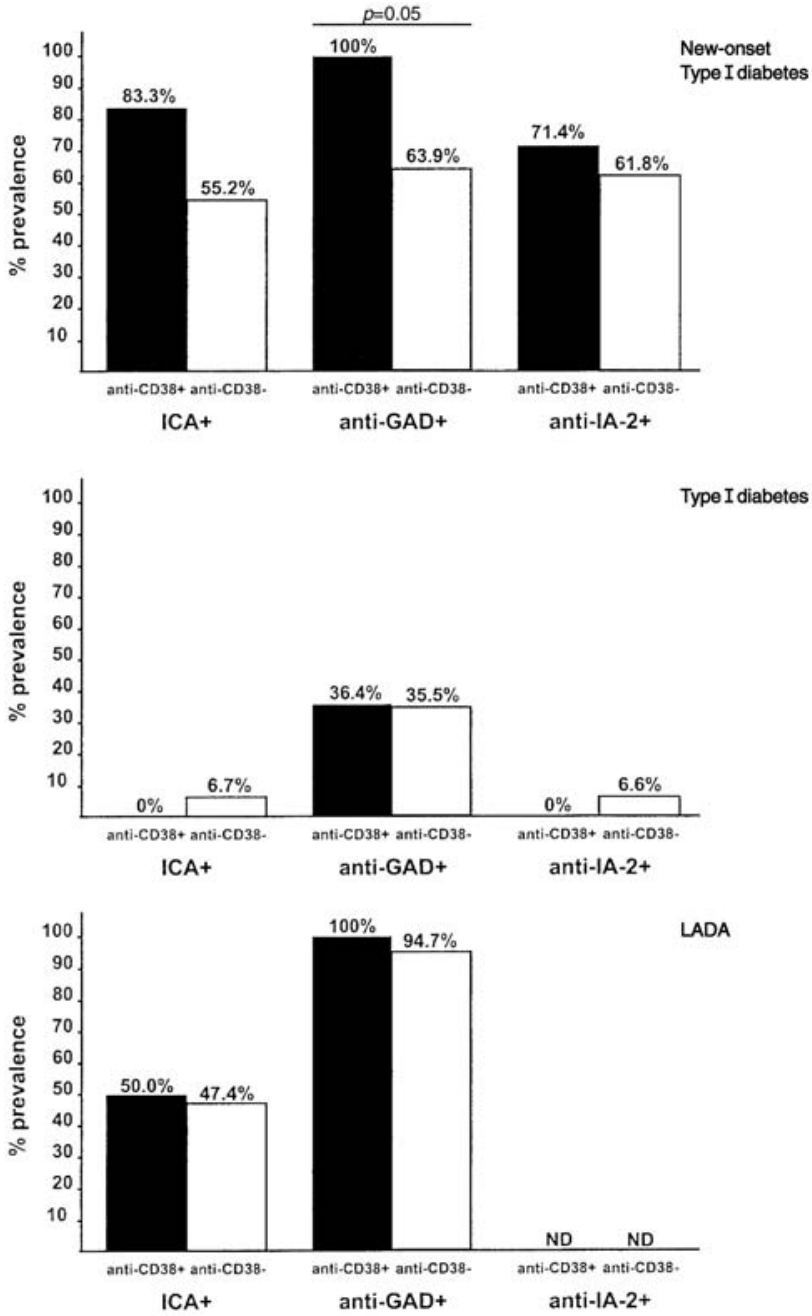

Fig. 3. Comparison of prevalences of conventional islet aAbs between anti-CD38+ (black bars) and anti-CD38- patients (white bars) in different diabetic groups. Per cent prevalence is given on top of each bar. ND, not determined

was found with higher frequency among new-onset Type I patients who were anti-CD $38^{+}$as compared to negative counterparts ( $100 \%$ vs $63.9 \% ; p=0.05$ ). A trend towards higher frequencies of ICA among anti-CD $38^{+}$subjects was also observed in this group $(83.3 \%$ vs $55.2 \% ; p=0.18)$. Among patients with longstanding Type I diabetes, where the prevalence of islet aAbs is much lower, anti-CD38 aAbs were not more prevalent among subjects positive or negative for other islet aAbs. No relevant association was found in the LADA cohort. As anti-IA-2 aAbs have a very low prevalence and little additional diagnostic value in LADA patients, being mainly associated to ICA and anti-GAD, they were not assayed in this group.

Lack of contribution of the anti-CD38 aAb fraction to the ICA reactivity. Among all the diabetic patients examined, only $37 \%$ of anti-CD $38^{+}$individuals were also $\mathrm{ICA}^{+}$. Given this rather incomplete overlapping 

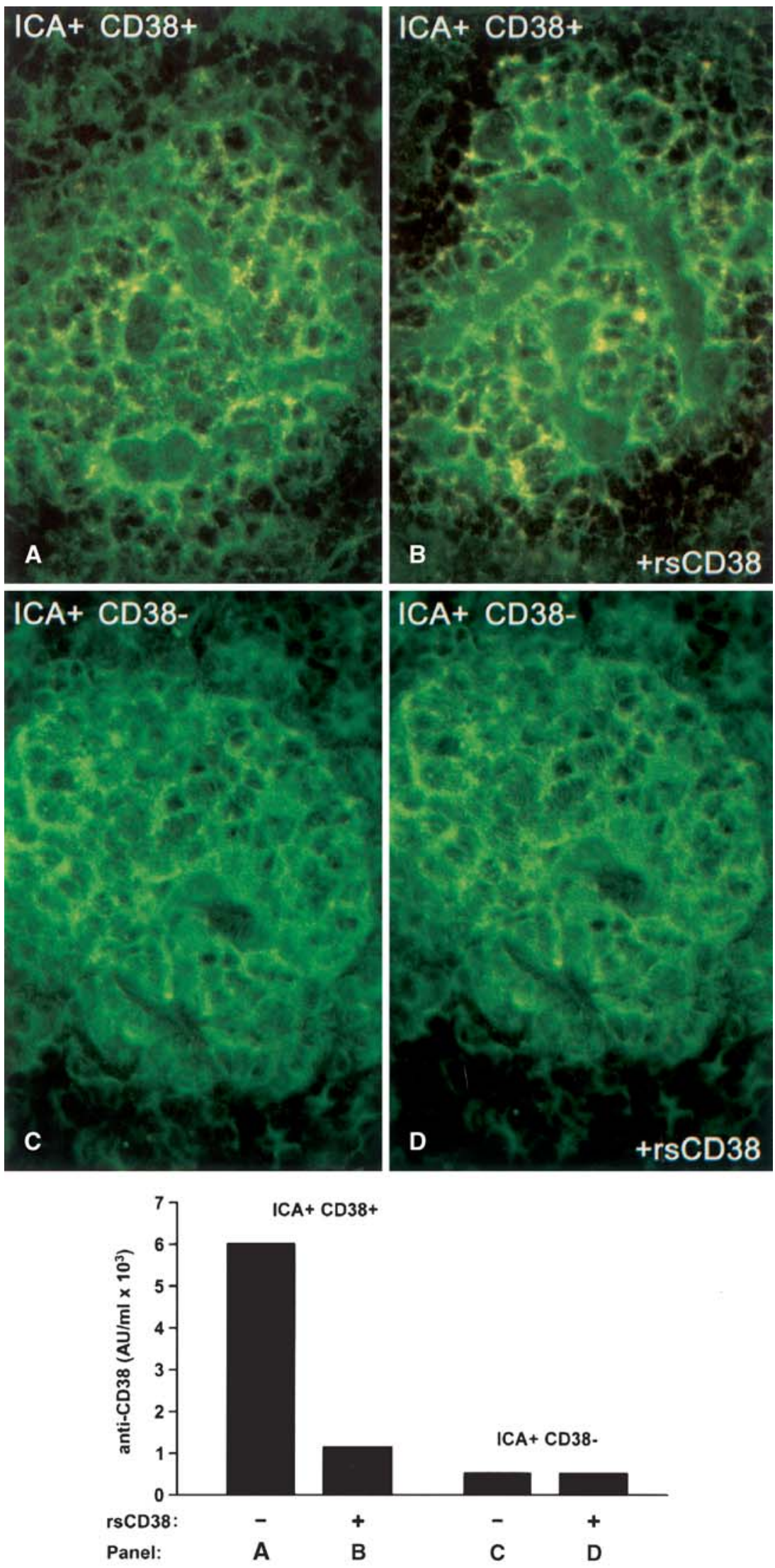

E 
between anti-CD38 aAbs and ICA, we investigated to further exclude any contribution of anti-CD38 aAbs to the ICA reactivity. To this aim, immunofluorescence for ICA on pancreatic tissue sections was carried out by preincubating different sera either with an irrelevant protein or with saturating amounts of rsCD38. As shown in Figure. 4, no difference in fluorescence intensity was evident when $\mathrm{ICA}^{+}$anti-CD38 ${ }^{+}$sera were preadsorbed with rsCD38 (Fig. 4, panel A, B). The same fluorescence intensity was also highlighted when $\mathrm{ICA}^{+}$anti-CD38- sera were used, either intact (Panel C) or preadsorbed with rsCD38 (Panel D). Preadsorption was indeed effective, since the procedure led to a consistent decrease in anti-CD38 reactivity in the anti-CD38+ sera, but not in the anti-CD38- ones (Panel E). ICA ${ }^{-}$anti-CD38+ sera did not give any signal (data not shown).

To exclude that these findings could be due to lack of CD38 expression in the human pancreas, immunofluorescence with a wide panel of anti-CD38 murine mAbs was carried out, but none of them gave a signal strong enough to be visualised on the autofluorescent background characteristic of pancreatic tissue sections (data not shown). This issue was therefore addressed by immunohistochemistry (Fig. 5): indeed, at low magnification (Panel A), islet cells were already visible as differentially stained by the AT1 mAb (antiCD38). On the contrary, exocrine pancreatic structures did not show any reactivity. Panel B shows a single islet of Langerhans at higher magnification. The signal visualised was specific, since no reactivity was detected when the AT1 mAb was first preadsorbed with saturating amounts of rsCD38 (Fig. 6, Panel B), as compared with the same $\mathrm{mAb}$ preincubated with an irrelevant protein (Panel A).

Agonistic effect of anti-CD38 aAbs on $\mathrm{Ca}^{2+}$ release. Among the anti-CD38 ${ }^{+}$sera identified in new-onset Type I patients and LADA patients, 8 out of $17(47.1 \%)$ anti-CD38+ samples for which sufficient serum was still available were analysed for their activity on $\mathrm{Ca}^{2+}$ release. As shown in Fig. 7, all the positive sera were capable of inducing $\mathrm{Ca}^{2+}$ release when incubated with $\mathrm{CD}^{+} 8^{+}$Jurkat $\mathrm{T}$ cells, thus showing agonistic activity, as previously reported [25]. On the contrary, none of the 21 control anti-

4 Fig. 4A-E. Comparison of ICA immunofluorescence before and after preadsorption with rsCD38. A $\mathrm{ICA}^{+}$anti-CD38 ${ }^{+}$serum preadsorbed with an irrelevant protein. $\mathbf{B}$ The same serum preadsorbed with rsCD38. C $\mathrm{ICA}^{+}$anti-CD38- serum preadsorbed with an irrelevant protein. D The same serum preadsorbed with rsCD38. E Anti-CD38 reactivity of the $\mathrm{ICA}^{+}$anti$\mathrm{CD}^{2} 8^{+}(\mathbf{A}, \mathbf{B})$ and $\mathrm{ICA}^{+}$anti-CD38- $(\mathbf{C}, \mathbf{D})$ sera used for ICA immunofluorescence in the corresponding panels, with $(+)$ or without (-) preadsorption with rsCD38. Results refer to a representative experiment carried out in triplicate with different $\mathrm{ICA}^{+}$anti-CD38 ${ }^{+}$and $\mathrm{ICA}^{+}$anti-CD38- sera
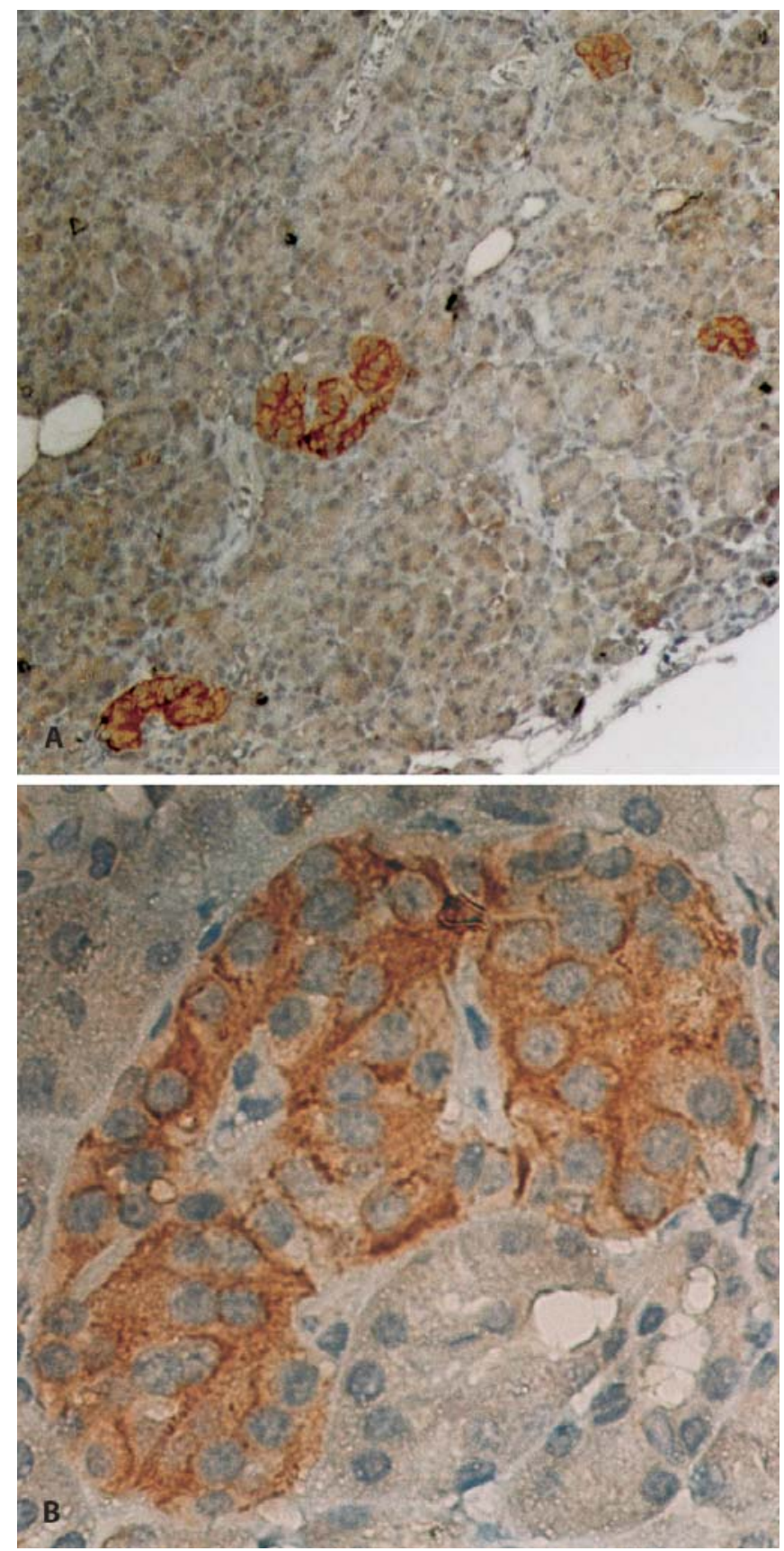

Fig. 5A, B. CD38 expression in the human pancreas, as assessed by immunohistochemical analysis on fixed sections with the AT1 anti-CD38 mAb and diaminobenzidine-peroxidase reaction. A Section of pancreatic tissue containing four islets of Langerhans, staining brown for CD38 $(\times 100)$. B Islet of Langerhans visualised at higher magnification $(\times 500)$

CD38- diabetic sera showed $\mathrm{Ca}^{2+}$-mobilising activity $(p<0.001)$.

Association of anti-CD38 aAb status with clinical and metabolic parameters. Anti-CD38 ${ }^{+}$and anti-CD38diabetic patients were compared for relevant clinical and metabolic characteristics. Despite the limited 


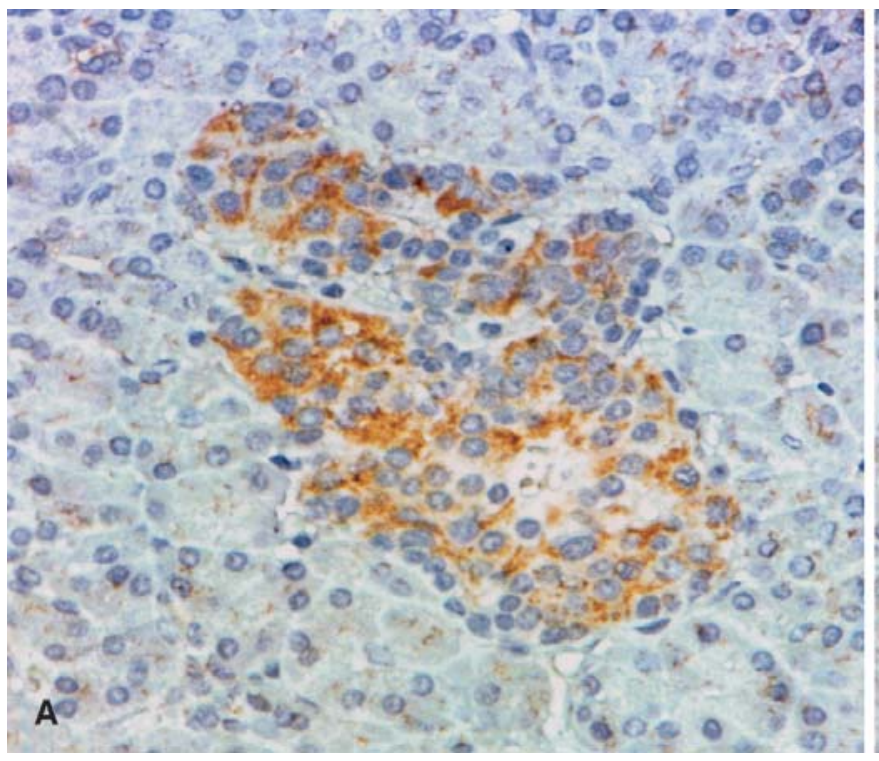

Fig. 6A, B. CD38 staining of pancreatic islets after preadsorption of AT1 anti-CD38 mAb $(\times 400)$. A Pancreatic islet reacted with the AT1 mAb preadsorbed with an irrelevant protein.

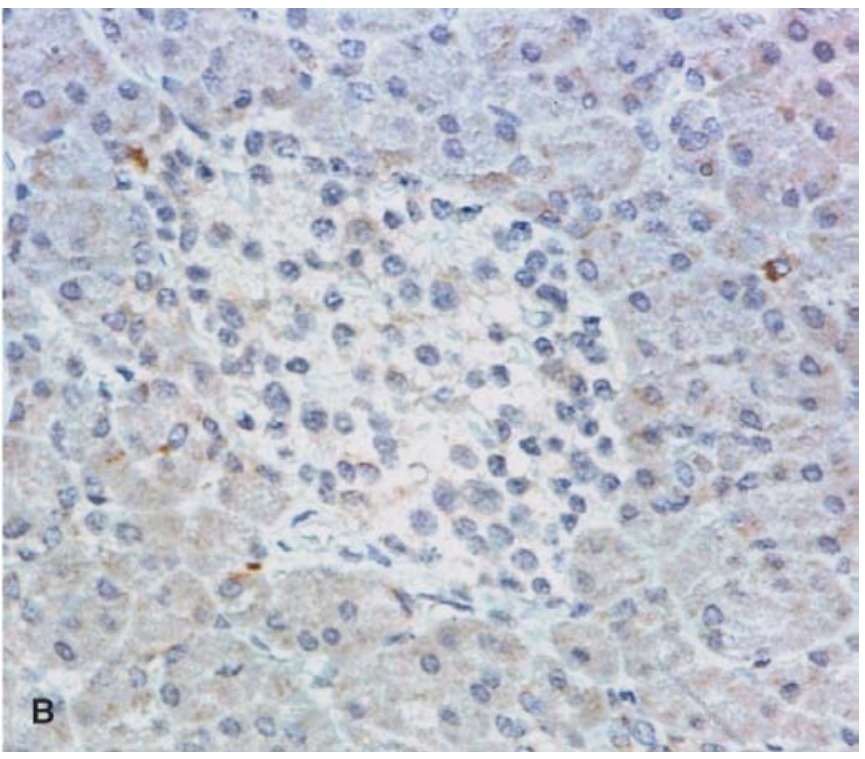

B The same islet reacted with the AT1 mAb preadsorbed with saturating amounts of rsCD38

Table 4. Comparison between clinical and metabolic characteristics of newly diagnosed Type I diabetic patients positive (anti$\mathrm{CD}^{+} 8^{+}$) and negative (anti-CD38-) for anti-CD38 autoantibodies

\begin{tabular}{llll}
\hline New-onset type I diabetic patients $(n=183)$ & Anti-CD38+ $(n=7)$ & Anti-CD38- $(n=176)$ & $p$ \\
\hline Sex (male and female) & $57.1 \%-42.9 \%$ & $54.5 \%-45.5 \%$ & $\mathrm{NS}$ \\
Age at diagnosis (years) & $15.9 \pm 7.1$ & $12.5 \pm 8.2$ & $\mathrm{NS}$ \\
Fasting plasma C-peptide $(\mathrm{ng} / \mathrm{ml})$ & $0.95(0.14-2.97)$ & $0.42(0-5.70)$ & 0.05 \\
Autoimmune diseases $(\%)$ & $0 \%$ & $2.8 \%$ & $\mathrm{NS}$ \\
\hline
\end{tabular}

Data are expressed as per cent proportion, means \pm SD or median (range), as appropriate

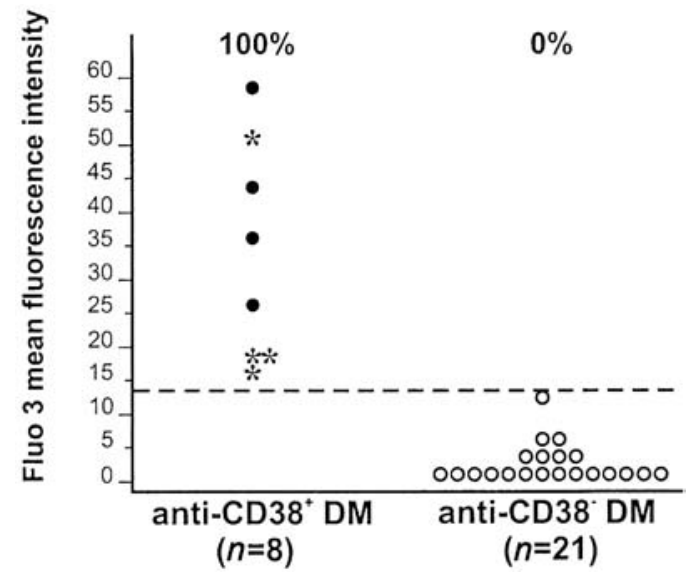

Fig. 7. Prevalence of agonistic activity among anti-CD38+ sera from new-onset Type I (O) patients and LADA (*) patients as opposed to anti-CD38- diabetic sera $(O)$. Values are expressed as the Fluo 3-AM mean fluorescence intensity; the dotted line indicates the cut-off value $(100 \%$ sensitivity and specificity) of 12.8 . The per cent proportion of anti-CD $38^{+}$ and control (anti-CD38-) sera showing agonistic properties is indicated above each distribution plot. $p<0.001$ for comparison between the two groups number of anti-CD38+ new-onset Type I diabetic patients identified $(n=7)$, these individuals were characterised by borderline significantly higher fasting plasma C-peptide values as compared to negative counterparts [median 0.95 (range $0.14-2.97$ ) vs 0.42 $(0-5.70) \mathrm{ng} / \mathrm{ml} ; p=0.05]$ (Table 4). Interestingly, this association was more evident when the analysis was limited to the anti-CD38+ sera showing agonistic activity $[1.14(0.50-2.97)$ vs $0.43(0-5.70) \mathrm{ng} / \mathrm{ml}$; $p=0.03$.

Also in the LADA cohort, the positivity for antiCD38 aAbs was associated with a trend towards higher fasting plasma C-peptide values [1.10 (0.08-5.98) vs $0.72(0-3.84) \mathrm{ng} / \mathrm{ml} ; p=0.35$ ] (Table 5). Also in this case, this trend was more effectively observed when only anti-CD $38^{+}$subjects showing agonistic aAbs were considered [1.62 (1.24-2.49) vs $0.72(0-5.98)$; $p=0.07]$. 
Table 5. Comparison between clinical and metabolic characteristics of LADA patients positive (anti-CD38 ${ }^{+}$) and negative (antiCD38-) for anti-CD38 autoantibodies

\begin{tabular}{lccc}
\hline LADA patients $(n=67)$ & Anti-CD38 $(n=10)$ & Anti-CD38- $(n=57)$ & $p$ \\
\hline Sex (male and female) & $40.0 \%-60.0 \%$ & $52.6 \%-47.4 \%$ & $\mathrm{NS}$ \\
Age at diagnosis (years) & $52.6 \pm 10.8$ & $48.7 \pm 11.0$ & $\mathrm{NS}$ \\
Diabetes duration (years) & $5(0-27)$ & $4(0-33)$ & $\mathrm{NS}$ \\
BMI $\left(\mathrm{kg} / \mathrm{m}^{2}\right)$ & $25.1 \pm 4.7$ & $24.9 \pm 3.9$ & $\mathrm{NS}$ \\
HbA $1 \%$ c & $8.6 \pm 2.4$ & $8.6 \pm 2.4$ & $\mathrm{NS}$ \\
Fasting plasma C-peptide (ng/ml) & $1.10(0.08-5.98)$ & $0.72(0-3.84)$ & $\mathrm{NS}$ \\
Therapy (D-OHA-I) & $10.0 \%-0 \%-90.0 \%$ & $12.3 \%-14.0 \%-73.7 \%$ & $\mathrm{NS}$ \\
Autoimmune diseases $(\%)$ & $22.2 \%$ & $25.6 \%$ & $\mathrm{NS}$ \\
\hline
\end{tabular}

Data are expressed as per cent proportion, means \pm SD or median (range), as appropriate. D, diet alone; OHA, oral hypoglycaemic agents; I, insulin (alone or in combination with $\mathrm{OHA}$ ).

\section{Discussion}

The significance of anti-CD38 aAbs as a marker of islet autoimmunity was addressed in this study by carrying out: (i) comparative analyses of anti-CD38 with conventional islet aAbs in different diabetic groups; (ii) in vitro functional studies of anti-CD38 aAbs and analysis of their associations with relevant clinical and metabolic parameters. Moreover, immunohistochemistry definitely showed that human pancreatic islets do express CD38, since previous reports only documented the presence of CD38 mRNA in this tissue, but not of the final protein product [41]. The fact that no signal was highlighted by immunofluorescence is probably due to the low epitopic density of pancreatic CD38, not enough to overwhelm the characteristic autofluorescence of this tissue [42, 43].

The overall body of data gathered indicates that anti-CD38 aAbs are new markers of beta-cell autoimmunity, characterised by their own unique features. Besides showing an independent pattern of expression among different diabetic groups as compared to the conventional islet aAbs, preadsorption experiments also showed that, in contrast to anti-GAD and antiIA-2 aAbs [8, 9, 11], anti-CD38 reactivity does not contribute to ICA immunofluorescence. This point is relevant as there are patients who are positive only for ICA but for neither anti-GAD nor anti-IA-2 [12, 21]: this additional ICA subspecificity is not as yet defined, and it does not correspond to anti-CD38 as well. The finding that anti-CD38 aAbs are not an ICA subfraction is not surprising if a key difference in the epitopes recognised is considered. Indeed, the ICA immunofluorescence is located in the cytoplasm [44], while CD38 is expressed on the cell surface; moreover, all the anti-CD38 aAbs selected so far recognize its extracellular portion, since the target rsCD38 used in our assay does not include the transmembrane and intracellular domains [34].

The choice of a single cut-off for anti-CD38 positivity, defined as the 97.5 centile $(6927 \mathrm{AU} / \mathrm{ml})$ of the values obtained in a wide cohort of healthy subjects, gives the advantage of uniforming the selection of positive cases in different study groups, thus avoiding the common influence of age on aAb titres [45]. Interestingly, the previously reported association of antiCD38 aAbs with higher residual beta-cell function in Type II diabetic patients [25] stands true even with these new selection criteria, both for fasting plasma C-peptide values [2.07 (0.54-8.27) vs $1.39(0-4.68)$ $\mathrm{ng} / \mathrm{ml} ; p=0.04]$ and insulin values [10.8 (5.5-140.3) vs $8.9(0.4-48.8) \mu \mathrm{U} / \mathrm{ml} ; p=0.04]$. Moreover, the fact that the anti-CD38 prevalence among LADA subjects was not higher than among long-standing Type I diabetic patients and Type II diabetic patients also confirms that, despite the autoimmune features, the previously characterised anti-CD38 ${ }^{+}$Type II diabetic subgroup does not correspond to LADA, as suggested by the different phenotype (i.e., higher residual insulin secretion, higher BMI) [25].

The precise diagnostic significance of anti-CD38 aAbs is still unclear. However, the observation that anti-CD38 aAbs were less prevalent at onset (3.8\%) than in long-standing disease $(11.7 \%)$ among Type I patients suggests that they could be a marker of a later stage of beta-cell destruction. In this respect, also the borderline association between anti-GAD and antiCD38 aAbs in new-onset Type I diabetes could be due to the longer persistence of anti-GAD as compared to the other islet aAbs. In our previous work [25], we did not find any correlation between anti-CD38 aAbs and disease duration among Type I diabetic patients, but the average long course of disease in that cohort made it difficult to address this issue. Despite the limit of the cross-sectional nature of this study, the increase in anti-CD38 prevalence with diabetes progression is of interest, since it represents a trend opposite to that of all the other islet aAbs, which show decreasing prevalences, although with different kinetics, as disease proceeds. However, longitudinal studies of Type I patients are needed to give a final answer to whether anti-CD38 arise at a later stage of beta-cell destruction.

The abundant expression of extra-pancreatic forms of CD38 could offer an explanation for the increase of 
anti-CD38 aAbs in long-standing disease. Indeed, islet aAbs commonly vanish a few years after the clinical onset of diabetes, when most beta cells have disappeared. Anti-GAD aAbs are the only exception, possibly because the GAD molecule is expressed also in nervous tissue, thus maintaining an antigenic trigger even after beta-cell failure; moreover, the short-lived aAbs can rapidly re-appear after islet transplantation [46]. A different time course of the primary immune trigger from the pancreas and subsequent persistence of antigenic stimuli from several tissues could justify the unique pattern of anti-CD38 aAbs.

Whether anti-CD38 aAbs are passive markers innocently witnessing beta-cell destruction or rather take an active part to the immune damage remains to be elucidated. Regarding this second possibility, the repeated observation that the vast preponderance of anti-CD38 aAbs are endowed with agonistic properties is appealing. Prolonged $\mathrm{Ca}^{2+}$ influx in the beta cell is known to trigger several deleterious effects such as desensitisation [4] and apoptosis [47]. The large preponderance of agonistic features for antiCD38 aAbs is also in line with the clinical and metabolic correlations observed. Indeed, the $\mathrm{Ca}^{2+}$-mobilising activity of anti-CD38 aAbs was shown not only to be effective in lymphocytic cell lines [25], but also in purified human islets, where it is coupled to stimulation of insulin secretion [30]. In this scenario, the observed in vitro agonistic features of anti-CD38 aAbs could be operating in vivo as well. Besides being previously reported in Type II diabetes [25], also anti-CD38+ subjects with new-onset Type I diabetes were characterised by higher fasting C-peptide values, and a stronger association was found when only agonistic anti-CD $38^{+}$sera were considered. A similar trend was also observed among LADA patients. Higher residual insulin secretion is a common trait when circulating anti-CD38 aAbs are found, with the only exception of long-standing Type I diabetes [25], where, expectedly, no target beta-cells are left for triggering by anti-CD38 aAbs. Whether this stimulating effect would be really beneficial in the long-term is opinable: triggering of insulin secretion would be initially favourable in the insulinopenic setting of early diabetes, but sustained stimulation could also add a further challenge and possibly lead to faster exhaustion of the limited beta-cell resources.

In conclusion, anti-CD38 aAbs are distinct markers of beta-cell autoimmunity, not merely accompanying the other islet aAbs but possibly marking a later stage of Type I diabetes. Moreover, they not only modify the in vitro behaviour of cells by triggering $\mathrm{Ca}^{2+} \mathrm{re}-$ lease and insulin secretion, but they could also affect the in vivo metabolic environment, since they identify subgroups of patients with better preserved beta-cell function. Longitudinal studies will further clarify the natural history of these aAbs and of their biological effects.
Acknowledgements. This work was supported by grants from MURST/COFIN, Rome, Italy (to PCP and FM). Juvenile Diabetes Research Foundation (JDRF) provided valuable help through its Islet Distribution Program. R. Mallone is a student at the Postgraduate School of Internal Medicine, University of Torino, Torino, Italy. E. Ortolan is a member of a Ph.D. program in Human Oncology. We gratefully acknowledge Dr. M. Cassader for C-peptide measurements. Parts of this study were presented as an oral communication at the 37 th Annual Meeting of the European Association for the Study of Diabetes, Glasgow, UK, 9-13 September 2001.

\section{References}

1. Atkinson MA, Eisenbarth GS (2001) Type 1 diabetes: new perspectives on disease pathogenesis and treatment. Lancet 358:221-229

2. Yki-Jarvinen H (1992) Glucose toxicity. Endocr Rev 13:415431

3. Unger RH, Zhou YT (2001) Lipotoxicity of beta-cells in obesity and in other causes of fatty acid spillover. Diabetes 50:S118-S121

4. Grill V, Bjorklund A (2001) Overstimulation and beta-cell function. Diabetes 50:S122-S124

5. Pratley RE, Weyer C (2001) The role of impaired early insulin secretion in the pathogenesis of Type II diabetes mellitus. Diabetologia 44:929-945

6. Wilkin TJ (2001) The accelerator hypothesis: weight gain as the missing link between Type I and Type II diabetes. Diabetologia 44:914-922

7. Bottazzo GF, Florin-Christensen A, Doniach D (1974) Islet cell antibodies in diabetes mellitus with autoimmune polyendocrine deficiencies. Lancet ii:1279-1283

8. Myers MA, Rabin DU, Rowley MJ (1995) Pancreatic islet cell cytoplasmic antibody in diabetes is represented by antibodies to islet cell antigen 512 and glutamic acid decarboxylase. Diabetes 44:1290-1295

9. Atkinson MA, Kaufman DL, Newman D, Tobin AJ, Maclaren NK (1993) Islet cell cytoplasmic autoantibody reactivity to glutamate decarboxylase in insulin-dependent diabetes. J Clin Invest 91:350-356

10. Verge CF, Stenger D, Bonifacio E et al. (1998) Combined use of autoantibodies (IA-2 autoantibody, GAD autoantibody, insulin autoantibody, cytoplasmic islet cell antibodies) in type 1 diabetes. Combinatorial Islet Autoantibody Workshop. Diabetes 47:1857-1866

11. Bonifacio E, Lampasona V, Genovese S, Ferrari M, Bosi E (1995) Identification of protein tyrosine phosphatase-like IA2 (islet cell antigen 512) as the insulin-dependent diabetes-related $37 / 40 \mathrm{~K}$ autoantigen and a target of islet-cell antibodies. J Immunol 155:5419-5426

12. Seissler J, Sonnaville JJ de, Morgenthaler NG et al. (1998) Immunological heterogeneity in Type I diabetes: presence of distinct autoantibody patterns in patients with acute onset and slowly progressive disease. Diabetologia 41:891897

13. Lohmann T, Seissler J, Verlohren H-J et al. (1997) Distinct genetic and immunological features in patients with onset of IDDM before and after age 40. Diabetes Care 20: 524-529

14. Seissler J, Hering B, Richter W et al. (1992) Antibodies to the $\mathrm{Mr} 64,000(64 \mathrm{~K})$ protein in islet cell antibody positive non-diabetic individuals indicate high risk for impaired beta-cell function. Diabetologia 35:550-554 
15. Christie MR, Tun RY, Lo SS et al. (1992) Antibodies to glutamic acid decarboxylase and tryptic fragments of islet $64 \mathrm{kD}$ antigen as distinct markers for the development of insulin-dependent diabetes: studies with identical twins. Diabetes 41:782-787

16. Christie MR, Genovese S, Cassidy D et al. (1994) Antibodies to islet $37 \mathrm{k}$ antigen, but not glutamate decarboxylase, discriminate rapid progression to IDDM in endocrine autoimmunity. Diabetes 43:1254-1259

17. Verge CF, Gianani R, Kawasaki E et al. (1996) Prediction of type I diabetes in first-degree relatives using a combination of insulin, GAD, and ICA512bdc/IA-2 autoantibodies. Diabetes 45:926-933

18. Groop LC, Bottazzo GF, Doniach D (1986) Islet cell antibodies identify latent type I diabetes in patients aged 35-75 years at diagnosis. Diabetes 35:237-241

19. Tuomi T, Groop LC, Zimmet PZ, Rowley MJ, Knowles W, Mackay IR (1993) Antibodies to glutamic acid decarboxylase reveal latent autoimmune diabetes mellitus in adults with a non-insulin-dependent onset of disease. Diabetes 42:359-362

20. Tuomi T, Carlsson A, Li H et al. (1999) Clinical and genetic characteristics of type II diabetes with and without GAD antibodies. Diabetes 48:150-157

21. Lohmann T, Kellner K, Verlohren H-J et al. (2001) Titre and combination of ICA and autoantibodies to glutamic acid decarboxylase discriminate two clinically distinct types of latent autoimmune diabetes in adults (LADA). Diabetologia 44:1005-1010

22. Pozzilli P, Di Mario U (2001) Autoimmune diabetes not requiring insulin at diagnosis (Latent Autoimmune Diabetes of the Adult). Diabetes Care 24:1460-1467

23. Ikehata F, Satoh J, Nata K et al. (1998) Autoantibodies against CD38 (ADP-ribosyl cyclase/cyclic ADP-ribose hydrolase) that impair glucose-induced insulin secretion in noninsulin-dependent diabetes patients. J Clin Invest 102:395-401

24. Pupilli C, Giannini S, Marchetti P et al. (1999) Autoantibodies to CD38 (ADP-ribosyl cyclase/cyclic ADP-ribose hydrolase) in Caucasian patients with diabetes: effects on insulin release from human islets. Diabetes 48:2309-2315

25. Mallone R, Ortolan E, Baj G et al. (2001) Autoantibody response to $\mathrm{CD} 38$ in Caucasian patients with type 1 and type 2 diabetes: immunological and genetic characterization. Diabetes 50:752-762

26. Ferrero E, Malavasi F (1999) The metamorphosis of a molecule: from soluble enzyme to the leukocyte receptor CD38. J Leukoc Biol 65:151-161

27. Mehta K, Malavasi F (2000) Human CD38 and related molecules. Karger, Basel

28. Takasawa S, Nata K, Yonekura H, Okamoto H (1993) Cyclic ADP-ribose in insulin secretion from pancreatic beta cells. Science 259:370-373

29. Okamoto H, Takasawa S, Nata K (1997) The CD38-cyclic ADP-ribose signalling system in insulin secretion: molecular basis and clinical implications. Diabetologia 40:14851491

30. Antonelli A, Baj G, Marchetti P et al. (2001) Human antiCD38 autoantibodies raise intracellular calcium and stimulate insulin release in human pancreatic islets. Diabetes 50:985-991
31. Bruno G, Merletti F, Biggeri A et al. (2001) Increasing trend of Type I diabetes in children and young adults in the province of Turin (Italy). Analysis of age, period and birth cohort effects from 1984 to 1996. Diabetologia 44:22-25

32. The Expert Committee on the Diagnosis and Classification of Diabetes Mellitus (2002) Report of the Expert Committee on the Diagnosis and Classification of Diabetes Mellitus. Diabetes Care 25:S5-S20

33. Alberti KGM, Zimmet PZ for the WHO Consultation (1998) Definition, diagnosis and classification of diabetes mellitus and its complications. Part 1: diagnosis and classification of diabetes mellitus. Provisional report of a WHO consultation. Diabet Med 15:539-553

34. Munshi CB, Fryxell KB, Lee HC, Branton WD (1997) Large-scale production of human CD38 in yeast by fermentation. Methods Enzymol 280:318-330

35. Borg H, Fernlund P, Sundkvist G (1997) Measurement of antibodies against glutamic acid decarboxylase 65 (GADA): two new 125I assays compared with [ $\left.{ }^{35} \mathrm{~S}\right] \mathrm{GAD}$ 65-ligand binding assay. Clin Chem 43:779-785

36. Rabin DU, Pleasic SM, Shapiro JA et al. (1994) Islet cell antigen 512 is a diabetes-specific islet autoantigen related to protein tyrosine phosphatases. J Immunol 152:31833188

37. Hagopian WA, Sanjeevi CB, Kockum I et al. (1995) Glutamate decarboxylase-, insulin-, and islet cell- antibodies and HLA typing to detect diabetes in a general populationbased study of Swedish children. J Clin Invest 95:15051511

38. Bonifacio E, Genovese S, Braghi S et al. (1995) Islet autoantibody markers in IDDM: risk assessment strategies yielding high sensitivity. Diabetologia 38:816-822

39. Dotta F, Falorni A, Tiberti C et al. (1997) Autoantibodies to the GM2-1 islet ganglioside and to GAD-65 at type 1 diabetes onset. J Autoimmun 10:585-588

40. Yokota I, Matsuda J, Naito E, Michinori I, Shima K, Kuroda Y (1998) Comparison of GAD and ICA512/IA-2 antibodies at and after the onset of IDDM. Diabetes Care 21:49-52

41. Takasawa S, Tohgo A, Noguchi N et al. (1993) Synthesis and hydrolysis of cyclic ADP-ribose by human leukocyte antigen CD38 and inhibition of the hydrolysis by ATP. J Biol Chem 268:26052-26054

42. Van de Winkle M, Maes E, Pipeleers D (1982) Islet cell analysis and purification by light scatter and autofluorescence. Biochem Biophys Res Commun 107:525-532

43. Jindal RM, McShane P, Gray DW, Morris PJ (1994) Isolation and purification of pancreatic islets by fluorescence activated islet sorter. Transplant Proc 26:653

44. Vives M, Somoza N, Soldevila G et al. (1992) Reevaluation of autoantibodies to islet cell membrane in IDDM. Failure to detect islet cell surface antibodies using human islet cells as substrate. Diabetes 41:1624-1631

45. Moulias R, Proust J, Wang A et al. (1984) Age-related increase in autoantibodies. Lancet i:1128-1129

46. Bach JF, Koutouzov S, van Endert PM (1998) Are there unique autoantigens triggering autoimmune diseases? Immunol Rev 164:139-155

47. Chandra J, Zhivotovsky B, Zaitsev S, Juntti-Berggren L, Berggren P-O, Orrenius S (2001) Role of apoptosis in pancreatic beta-cell death in diabetes. Diabetes 50:S44-S47 